\title{
A Vascular Malformation Presenting as a Peripheral Nerve Sheath Tumor
}

\author{
Vikas Parmar $^{1}$ Clayton Haldeman ${ }^{1}$ Steve Amaefuna ${ }^{1}$ Amgad S. Hanna ${ }^{1}$ \\ ${ }^{1}$ Department of Neurological Surgery, University of Wisconsin, \\ Madison, Wisconsin, United States \\ J Brachial Plex Peripher Nerve Inj 2016;11:e38-e41.

\begin{abstract}
Address for correspondence Amgad S. Hanna, MD, Department of Neurological Surgery, University of Wisconsin Hospitals and Clinics, 600 Highland Ave., K4/822, Madison, WI 53705, United States (e-mail: a.hanna@neurosurgery.wisc.edu).
\end{abstract}

\author{
Abstract \\ Keywords \\ - brachial plexus \\ - nerve sheath tumor \\ - peripheral nerve \\ schwannoma \\ - venous malformation
}

\section{Case Presentation}

A 50-year-old man presented with a left axillary mass that had been palpable for the previous 2 years. He had experienced pain in the left shoulder and chest wall area for the previous 3 years. He reported his pain was 5 to 6 out of 10 on the visual analog scale. He denied any weakness, numbness, or tingling of the arm.

Physical exam revealed a firm, ovoid mass on the inside of the left upper arm. The mass was slightly tender to palpation. The mass was more mobile in the horizontal direction than the vertical. The overlying skin looked normal with no discoloration or discharge. No other swellings were noted in the body. We elicited a positive Tinel sign with ulnar distribution tingling. Physical exam otherwise was unremarkable.

Magnetic resonance imaging (MRI) with and without contrast demonstrated a T1 hypointense, T2 hyperintense homogeneously enhancing mass within the left upper arm (-Fig. 1). The mass was mildly lobulated, but well circumscribed, and measured $4.2 \mathrm{~cm}$ craniocaudal $\times 2.1 \mathrm{~cm}$ transverse $\times 2.2 \mathrm{~cm}$ anteroposterior. It was located between the triceps and coracobrachialis/biceps muscles, just posteri-

\author{
received \\ April 8, 2016 \\ accepted
}

August 14, 2016 or to the neurovascular bundle of the upper extremity. It appeared that the mass was arising exophytically from the ulnar nerve, and it was presumed to be a schwannoma.

We elected to remove the mass given the patient's pain. The median, ulnar, and medial antebrachial cutaneous nerves, as well as the brachial artery, were identified during our dissection (-Fig. 2). There was no nerve that was contributing to the mass. A branch of the brachial vein, however, was seen entering the mass at the more cephalad end. This was carefully dissected and appeared to be a neck. At this point, we identified that this mass appeared to be a venous malformation (VM). Thus, the contributing vein was ligated and the VM was removed. Gross examination of a cross-section revealed blood clots. Histology confirmed a VM and demonstrated no nervous tissue (-Fig. $\mathbf{3}$ ).

\section{Discussion}

Venous Malformations, thin-walled vascular dilations of various sizes, are the most common type of vascular malformation. The incidence of VM is estimated to be between $1 / 10,000$ and $1 / 5,000$ and the prevalence is thought to be
License terms

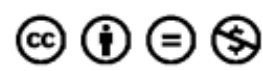

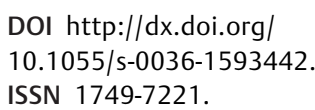

DOI http://dx.doi.org/ 10.1055/s-0036-1593442 ISSN $1749-7221$.

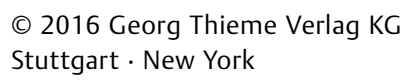
Stuttgart · New York

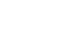

We present the case of a venous malformation (VM) masquerading as a schwannoma. compressible, blue masses that are associated with pain or dysesthesia. VMs are commonly found in the head and neck as well as the distal extremities. Notably, imaging, and enhance markedly with contrast. However, VMs tend to be poorly and sclerotherapy are the primary treatment options. In this case report, we present a unique in that the patient presented with symptoms and imaging qualities characteristic for a peripheral nerve schwannoma. 

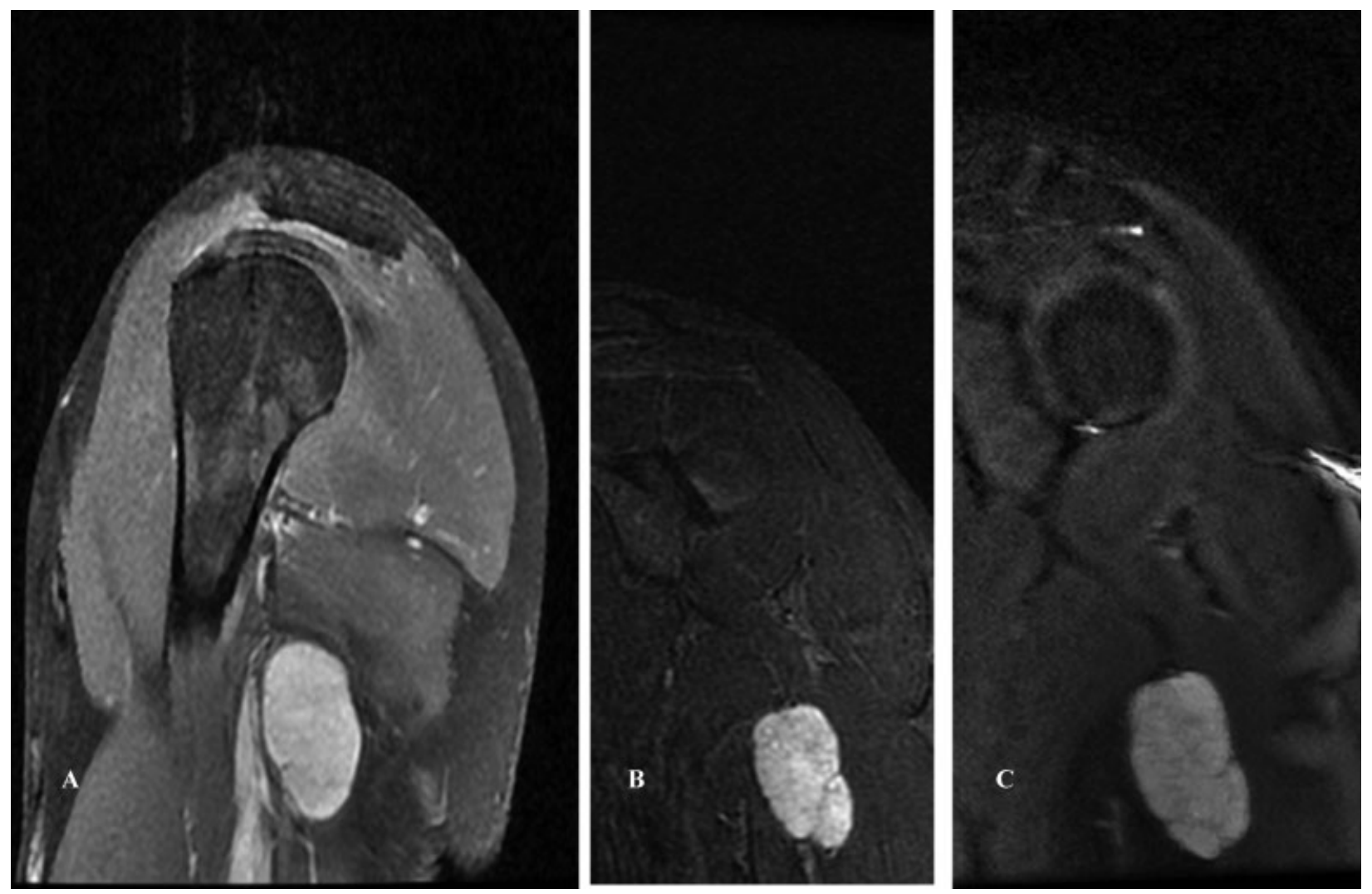

Fig. 1 (A) Sagital T1 with contrast, (B) coronal STIR (short inversion time inversion recovery), and (C) coronal T2-weighted MRI sequences show that the mass is $\mathrm{T} 2$ hyperintense and enhances homogenously.

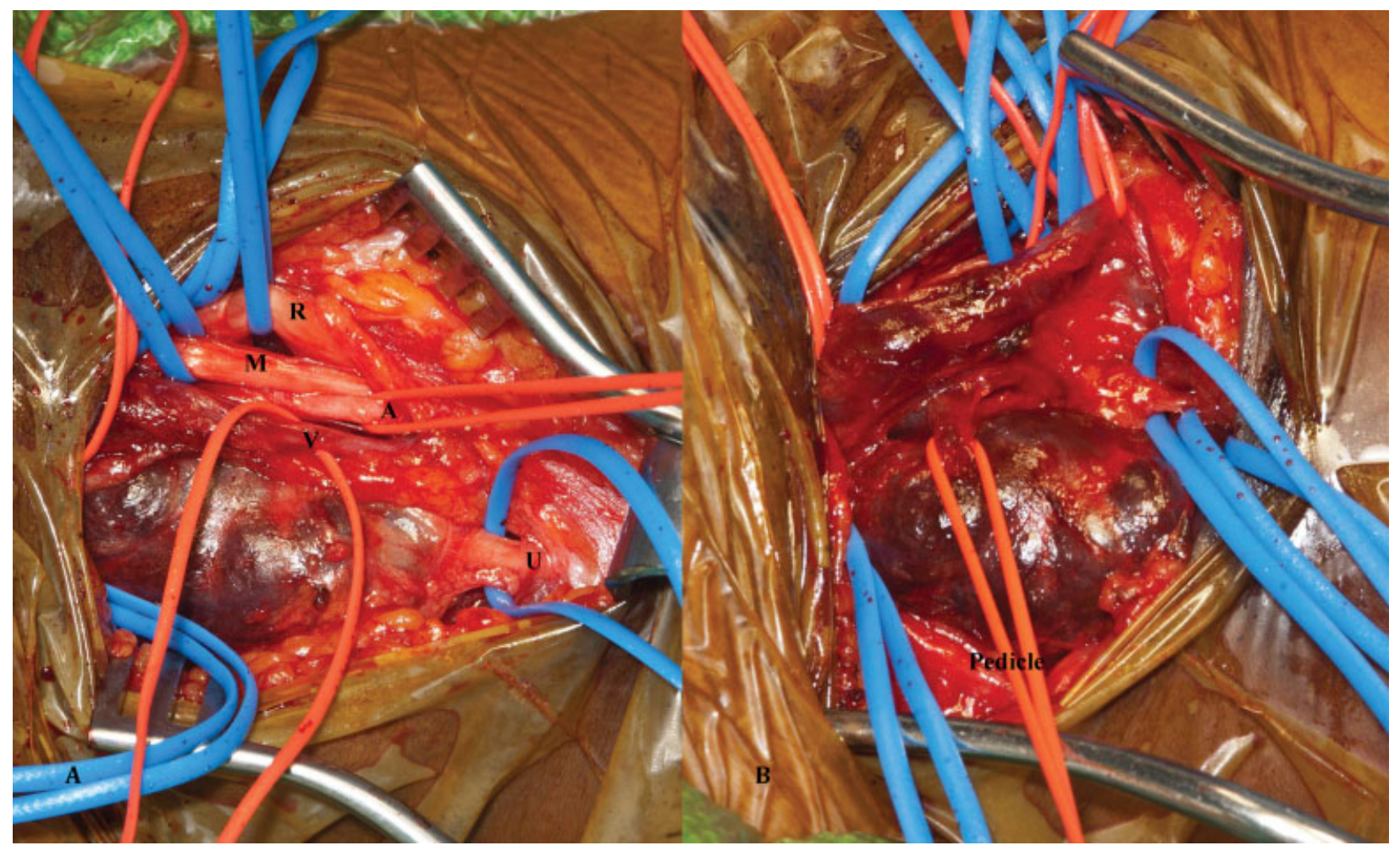

Fig. 2 (A, B) Dissection of the mass showing radial $(R)$, median $(M)$, ulnar nerves $(U)$, artery $(A)$, and vein $(V)$ along the hemangioma and the pedicle. 


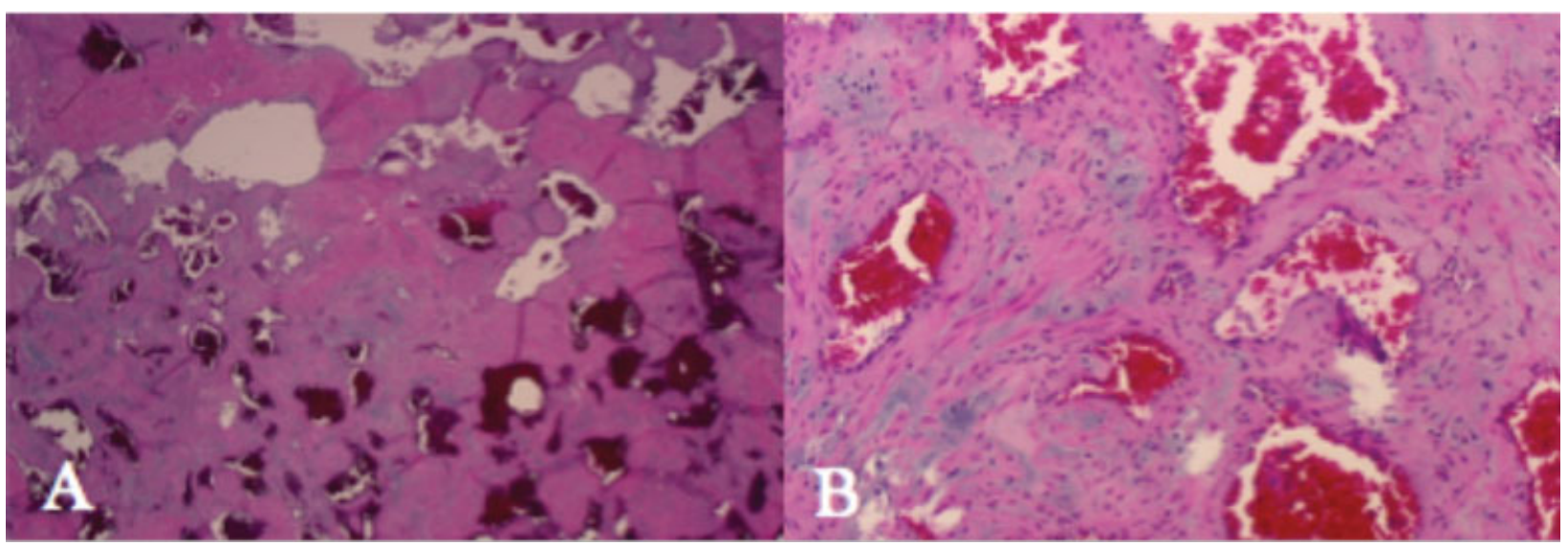

Fig. 3 (A) The excised tissue consists entirely of small-medium-sized thinned-wall vascular channels containing red blood cells. (B) Magnified view demonstrates no prominent cellular atypia, necrosis, and/or mitoses. Nervous tissue is also not present.

approximately $1 \%{ }^{1,2}$ Though all are present at birth, the most rapid growth occurs during puberty, and they continue to grow throughout life; only $50 \%$ are identified at birth. ${ }^{3}$ In this respect, they differ from hemangiomas. VMs can appear superficial, deep, diffuse, localized, or, rarely, as multiple lesions. They can occur in any tissue in the body. The most common locations reported are in the head and neck (40\%), followed by the extremities (40\%) and trunk (20\%). ${ }^{4}$ Patients with VMs present as soft, blue masses that are compressible; the most common complaint is pain.

Proper workup for underlying coagulopathy must follow a diagnosis of VM. Various genetic syndromes, such as Kassabach-Merritt syndrome, Servelle-Martorell syndrome, Klippel-Trénaunay syndrome, and Parkes Weber syndrome, have been associated with vascular malformations among other pathologies and must be excluded.

MRI has become the modality of choice for imaging VMs. Slow-flow VMs are typically hypointense on T1-weighted imaging, have bright signal intensity on $\mathrm{T} 2$, and enhance markedly with contrast. ${ }^{5}$ Very often, large VMs have obvious phleboliths (dark circular areas) and poorly defined venous lakes on $\mathrm{T} 2$ imaging.

Indications for treatment for VMs primarily include pain and functional impairment, or are cosmesis related. Elastic therapy, sclerotherapy, and surgical resection are the potential therapeutic options. Elastic compression stockings have been shown to limit the swelling and improve coagulopathy. ${ }^{6}$ In fact, in one series of 121 successfully treated VMs, $8 \%$ had no treatment and $24 \%$ had aspirin and compression garments. ${ }^{3}$ Sclerotherapy, on the other hand, is the mainstay treatment for diffuse VMs that involve multiple muscle groups; sclerotherapy injection of absolute ethanol induces inflammation and obliteration of affected veins. Injections are usually bimonthly until the malformations shrink. Success rate in the largest series of 87 patients treated with an average of three sessions over 8 months was $95 \%$; another series reports a success rate of $76 \%{ }^{7,8}$ Complications reported include blistering, full-thickness cutaneous necrosis, and nerve injury. ${ }^{7}$ Surgical resection is reserved for VMs localized to single muscle or muscle group or causing neurologic impairment. In the Enjolras et al series, 6 of 11 upper extremity VMs were treated with surgical skin excision with or without muscle excision. Outcomes were reported as bad (1/6), unchanged (2/6), mediocre (1/6), and improved (2/6). ${ }^{6}$

The focus we would like to draw in this article, however, is a comparison with peripheral nerve schwannomas (PNSs). Of note, PNSs also present with a palpable mass (96\%) often associated with referred dysesthesia (95\%) and similarly, on MRI sequencing, are isotense or hypointense to muscle on T1 and hyperintense on T2. ${ }^{8}$ They also vividly enhance with contrast. Typically, intramuscular VMs can be differentiated from PNSs based on poorly circumscribed venous lakes and phleboliths.

Table 1 MRI and physical exam differences between PNS and VM

\begin{tabular}{|l|l|l|}
\hline & Peripheral nerve schwannoma & Venous malformation \\
\hline Symptoms & Dysesthesia & Pain \\
\hline Physical exam & Palpable mass, Tinel sign & Palpable, blueish, compressible mass \\
\hline MRI findings & $\begin{array}{l}\text { T1: isointense/hypointense; T2: hyperintense } \\
\text { Typically not lobulated, but well circumscribed } \\
\text { Closely adhering to a specific nerve } \\
\text { Fusiform appearance with demonstrable } \\
\text { inlet and outlet nerve }\end{array}$ & $\begin{array}{l}\text { Fast-flowing T1: hyperintense; T2: hyperintense } \\
\text { Slow-flowing T1: hypointense; T2: hyperintense } \\
\text { Lobulated with venous lakes, poorly circumscribed } \\
\text { Phleboliths present } \\
\text { Mottled appearance }\end{array}$ \\
\hline
\end{tabular}

Abbreviation: MRI, magnetic resonance imaging. 
Simon et al demonstrated that high-resolution ultrasonography (HRUS) correctly differentiated tumor and motor fascicles when compared with intraoperative electrophysiologic monitoring. ${ }^{9}$ Differentiating between PNS and VM with HRUS is difficult as blood can often be stagnant in slow-flow VMs. However, Zardi et al demonstrate the benefit of power flow sonography in identifying "red" and "blue" vascular signal spots that indicate vascularity which is present in PNSs but absent in VMs. ${ }^{9}$ Likewise, VMs will demonstrate compressibility given even light pressure from a US scanner head, whereas PNS are not compressible with scanner head pressure. MRI with tractography demonstrated good correspondence with both HRUS and intraoperative electrophysiologic monitoring. ${ }^{10}$ This may be useful for differentiating PNS and VM.

Primary treatment for a PNS is complete surgical resection, whereas that for a VM is conservative therapy with or without sclerotherapy. ${ }^{11}$ As peripheral nerve surgeons who have limited exposure to peripheral vascular anomalies, we must be wary of a slow-flowing VM in our differential for an upper extremity schwannoma. We recommend being cognizant to the physical exam and minute details in MRI, differences of which are demonstrated in - Table 1. Alternatively, a trial of conservative compressive therapy with aspirin for a few weeks for suspicious masses may elucidate this differential. However, we do not recommend this approach as aspirin may postpone surgery and, more importantly, painful, peripheral masses could very well be a malignant peripheral nerve sheath tumor, which mandate early resection.

\section{References}

1 Eifert S, Villavicencio JL, Kao TC, Taute BM, Rich NM. Prevalence of deep venous anomalies in congenital vascular malformations of venous predominance. J Vasc Surg 2000;31(3):462-471

2 Puig S, Casati B, Staudenherz A, Paya K. Vascular low-flow malformations in children: current concepts for classification, diagnosis and therapy. Eur J Radiol 2005;53(1):35-45

3 Hein KD, Mulliken JB, Kozakewich HP, Upton J, Burrows PE. Venous malformations of skeletal muscle. Plast Reconstr Surg 2002; 110(7):1625-1635

4 Kransdorf MJ, Murphey MD. Imaging of Soft Tissue Tumors. 2nd ed. Philadelphia, PA: Lippincott Williams \& Wilkins; 2006

5 Walker EA, Fenton ME, Salesky JS, Murphey MD. Magnetic resonance imaging of benign soft tissue neoplasms in adults. Radiol Clin North Am 2011;49(6):1197-1217, vi

6 Enjolras O, Ciabrini D, Mazoyer E, Laurian C, Herbreteau D. Extensive pure venous malformations in the upper or lower limb: a review of 27 cases. J Am Acad Dermatol 1997;36 (2, Pt 1):219-225

7 Lee BB, Do YS, Byun HS, Choo IW, Kim DI, Huh SH. Advanced management of venous malformation with ethanol sclerotherapy: mid-term results. J Vasc Surg 2003;37(3):533-538

8 Ogose A, Hotta T, Morita T, et al. Tumors of peripheral nerves: correlation of symptoms, clinical signs, imaging features, and histologic diagnosis. Skeletal Radiol 1999;28(4):183-188

9 Zardi EM, Vadalà G, Buzzulini F, et al. Imaging and surgical approach for a schwannoma of the hand. J Med Ultrason (2001) 2014;41(2):229-232

10 Simon NG, Cage T, Narvid J, Noss R, Chin C, Kliot M. High-resolution ultrasonography and diffusion tensor tractography map normal nerve fascicles in relation to schwannoma tissue prior to resection. J Neurosurg 2014;120(5):1113-1117

11 Berenguer B, Burrows PE, Zurakowski D, Mulliken JB. Sclerotherapy of craniofacial venous malformations: complications and results. Plast Reconstr Surg 1999;104(1):1-11, discussion 12-15 